\title{
A Comparative Analysis of Assignment Problem
}

\author{
${ }^{1}$ SHWETA SINGH, ${ }^{2}$ G.C. DUBEY, ${ }^{3}$ RAJESH SHRIVASTAVA \\ ${ }^{1}$ Department of Mathematics Radharaman Institute of Tech. \& Science, Bhopal (M.P.) \\ ${ }^{2}$ Department of Mathematics Govt M.G.M. College Itarsi (M.P.) \\ ${ }^{3}$ Department of Mathematics Govt Benazir College, Bhopal (M.P.)
}

\begin{abstract}
Assignment problems arise in different situation where we have to find an optimal way to assign nobjects to m-other objects in an injective fashion. The assignment problems are a well studied topic in combinatorial optimization. These problems find numerous application in production planning, telecommunication VLSI design, economic etc. The assignment problems is a special case of Transportation problem. Depending on the objective we want to optimize, we obtain the typical assignment problems. Assignment problem is an important subject discussed in real physical world we endeavor in this paper to introduce a new approach to assignment problem namely, matrix ones assignment method or MOA -method for solving wide range of problem. An example using matrix ones assignment methods and the existing Hungarian method have been solved and compared it graphically. Also some of the variations and some special cases in assignment problem and its applications have been discussed in the paper.
\end{abstract}

Keywords: - Assignment problem, Hungarian assignment method (HA) method, Linear Integer Programming, Matrix ones assignment method (MOA) method, optimization

\section{INTRODUCTION}

Assignment problems deals with the question how to assign $n$ objects to $m$ other objects in an injective fashion in the best possible way. An assignment problem is completely specified by its two components the assignments, which represent the underlying combinatorial structure, and the objective function to be optimized, which models "the best possible way".The assignment problem refers to another special class of linear programming problem where the objective is to assign a number of resources to an equal number of activities on a one to one basis so as to minimize total costs of performing the tasks at hand or maximize total profit of allocation. In other words, the problems is, how should the assignment be made so as to optimize the given objective. Different methods have been presented for assignment problem and various articles have been published on the see [1], [2] and [3] for the history of these methods.

\section{Few Application of the Assignment Method :}

1. Assign sales people to sales territories.

2. Assign Vehicles to routes.

3. Assign accountants to client accounts.

4.Assign contracts to bidders through systematic evaluation of bids from competing suppliers.

5. Assign naval vessels to petrol sectors.

6. Assign development engineers to several construction sites.

7. Schedule teachers to classes etc.

8. Men are matched to machines according to pieces produced per hour by each individual on each machine.

9. Teams are matched to project by the expected cost of each team to accomplish each project.

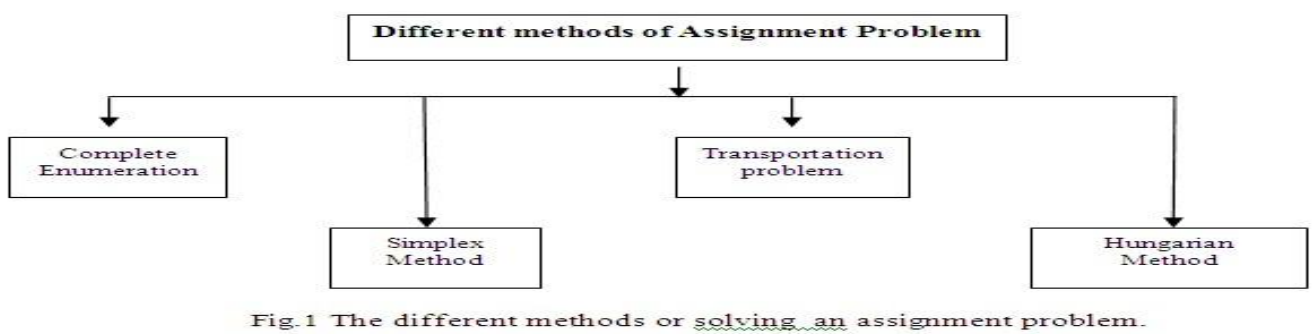

Tote:- It may be noted that assignment problem is a variation of transportation problem with two always be such that there would be only one assignment in a given row or column of the cost matrix. 


\section{Approach of the Assignment Model:}

Each assignment problem has a table or matrix associated with it. Generally the row contain the objects or people we wish to assign, and the column comprise the jobs or task we want them assigned to. Consider a problem of assignment of $\mathrm{n}$ resources to $\mathrm{m}$ activities so as to minimize the overall cost or time in such a way that each resource can associate with one and only one job. The cost matrix $\left(\mathrm{C}_{\mathrm{ij}}\right)$ is given as under :

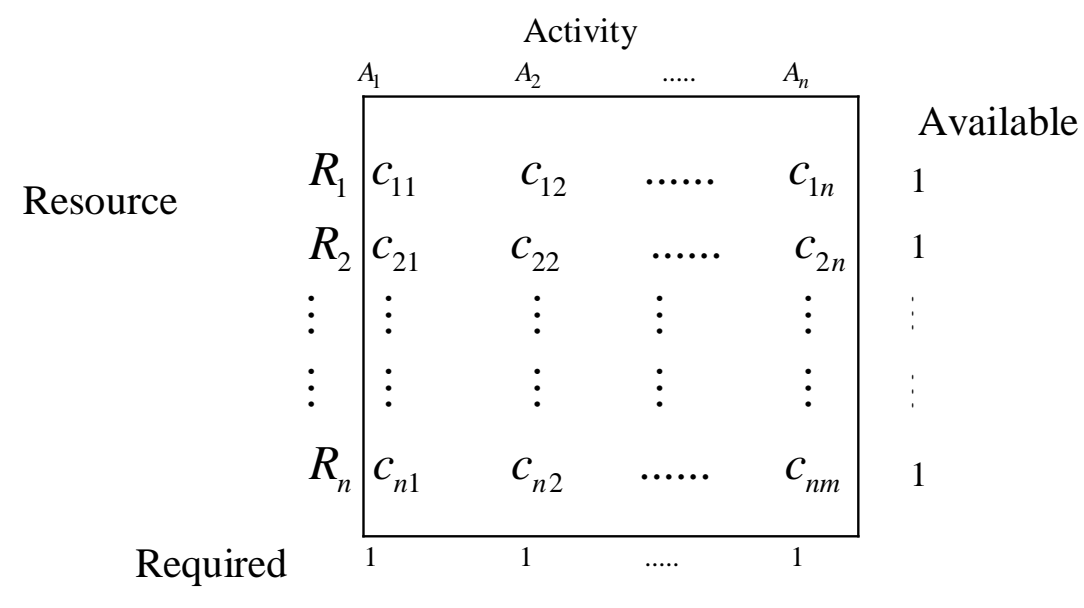

The cost matrix is same as that of a T.P except that availability at each of the resource and the requirement at each of the destinations is unity.

Let $\mathrm{x}_{\mathrm{ij}}$ denote the assignment of $\mathrm{i}^{\text {th }}$ resource to $\mathrm{j}^{\text {th }}$ activity, such that

$$
x_{i j}= \begin{cases}1 & ; \text { if resource } \mathrm{i} \text { is assigned to activity } \mathrm{j} \\ 0 & ; \text { otherwise }\end{cases}
$$

Then the mathematical formulation of the assignment problems is

Minimize $\mathrm{z}=\sum_{i=1}^{n} \sum_{j=1}^{n} c_{i j} x_{i j}$

Subject to the constraints

$$
\sum_{i=1}^{n} x_{i j}=1 \text { and } \sum_{j=1}^{n} x_{i j}=1: x_{i j}=0 \text { or } 1
$$

For all $\mathrm{i}=1,2, \ldots \mathrm{n} \quad$ \&

$$
\mathrm{j}=1,2 \ldots \mathrm{n}
$$

III. METHODS OF ASSIGNMENT PROBLEM:

Food's technique method or Hungarian Assignment method (Minimization Case)

The Hungarian method (also known as Flood's Technique or the Reduced Matrix method) of assignment provides us with an efficient means of finding the optimal solutions without having to make a direct comparsion of every option. It operates on a principle of matrix reduction. This just means that by subtracting and adding appropriate numbers in the cost table or matrix, we can reduce the problem to a matrix of opportunity costs (opportunity costs show the relative penalties associated with assigning any worker to a job as opposed to making the best or least cost assignment). If we can reduce the matrix to the point where there is one zero element in each row and column, it will then be possible to make optimal assignment, i.e. assignment in which all the opportunity costs are zero. Hungarian method of assignment problem (minimization case) can be summarized in the following steps:

Steps1. Find the opportunity cost table by:

a. Subtracting the smallest number in each row of the original cost table or matrix from every number in that row and.

b. Then subtracting the smallest number in each column of the table obtained in part (a) from every number in that column. 
2. Make assignments in the opportunity cost matrix in the following way:

a. Examine the rows successively until a row with exactly one unmarked zero is found. Enclose this zero in a box ( $\square$ ) as an assignment will be made there and cross ( $\mathrm{x}$ ) all other zeros appearing in the corresponding column as they will not be considered for future assignment. Proceed in this way until all the rows have been examined.

b. After examining all the rows completely, examine the columns successively until a column with exactly one unmarked zero is found. Make an assignment to this single zero by putting square $(\square)$ around it and cross out (x) all other zeros appearing in the corresponding row as they will not be used to make any other assignment in that row, Proceed in this manner until all columns have been examined.

c. Repeat the operations (a) and (b) succesively until one of the following situations arises.:

i. All the zeros in rows/columns are either marked $(\square)$ or corssed $(\times)$ and there is exactly one assignment in each row and in each column. In such a case optimal assignment policy for the given problem is obtained.

ii. there may be some row (or column) without assignment, i.e., the total number of marked zeros is less than the order of the matrix. In such a case, proceed to next Step 4.

3. Revise the opportunity cost table: Draw the minimum number of vertical and horizontal lines necessary to cover all the zeros in the reduced cost table obtained from Step 2 by adopting the following procedure:

i. $\quad$ Mark $(\sqrt{ })$ all rows that do not have assignments.

ii. $\quad$ Mark $(\sqrt{ })$ all columns (not already marked) which have zeros in the marked rows [step 4 (ii)].

iii. Mark $(\sqrt{ })$ all rows (not already marked) that have assignments in marked columns [step 4(iii)].

iv. Repeat steps 4(ii) and (iii) until no more rows or cloumns can be marked.

v. Draw straight lines through each unmarked row and each marked column.

If the number of lines drawn (or total assignments) is equal to the number of rows (or columns) then the current solution is the optimal solution, otherwise go to Step 4 .

\section{Develop the new revised opportunity cost table:}

An optimal solution is seldom obtained from the intitial opportunity cost table. Often, we will need to revise the table in order to shift one (or more) of the zero costs from its present location (covered by lines) to a new uncovered location in the table. Intuitively, we would want this uncovered location to emerge with a new zero opportunity cost. This is accomplished by subtracting the smallest number not covered by a line from all numbers not covered by a straight line. This same smallest number is then added to every number (including zeroes) lying at the intersection of any two lines.

5. Repeat Steps 2 to 4 until an opitmal solution is obtained.

Numerical example using Hungarian method

\section{Row Reduction}

\begin{tabular}{|c|c|c|c|c|}
\hline 1 & 2 & 3 & 4 & 5 \\
\hline \begin{tabular}{l|l|l}
12
\end{tabular} & 8 & 7 & 15 & 4 \\
\hline \begin{tabular}{l|l}
2 & 7
\end{tabular} & 9 & 1 & 14 & 10 \\
\hline \begin{tabular}{l|l|l}
3 & 9
\end{tabular} & 6 & 12 & 6 & 7 \\
\hline $4 \mid 7$ & 6 & 14 & 6 & 10 \\
\hline 59 & 6 & 12 & 10 & 6 \\
\hline
\end{tabular}

\section{Column Reduction}

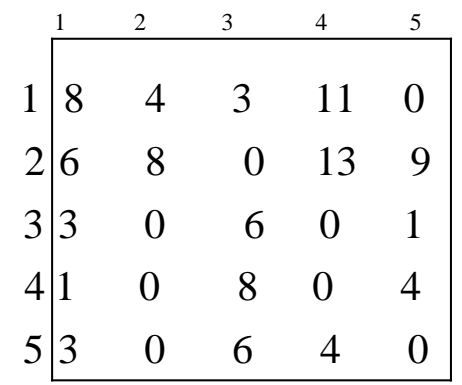




\section{Zero Assignment}

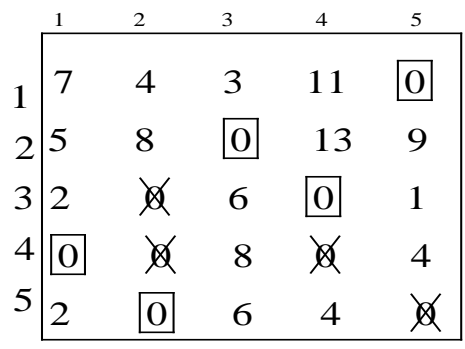

Since from the last table we see that all the zeros are either assigned or crossed out i.e. the total assigned zero's is $5=$ to the n (no of row \& columns 5) therefore from the example we get $(1,5),(2,3)(3,4)$ $(4,1)(5,2)$ and minimum cost is 24 using HA method-

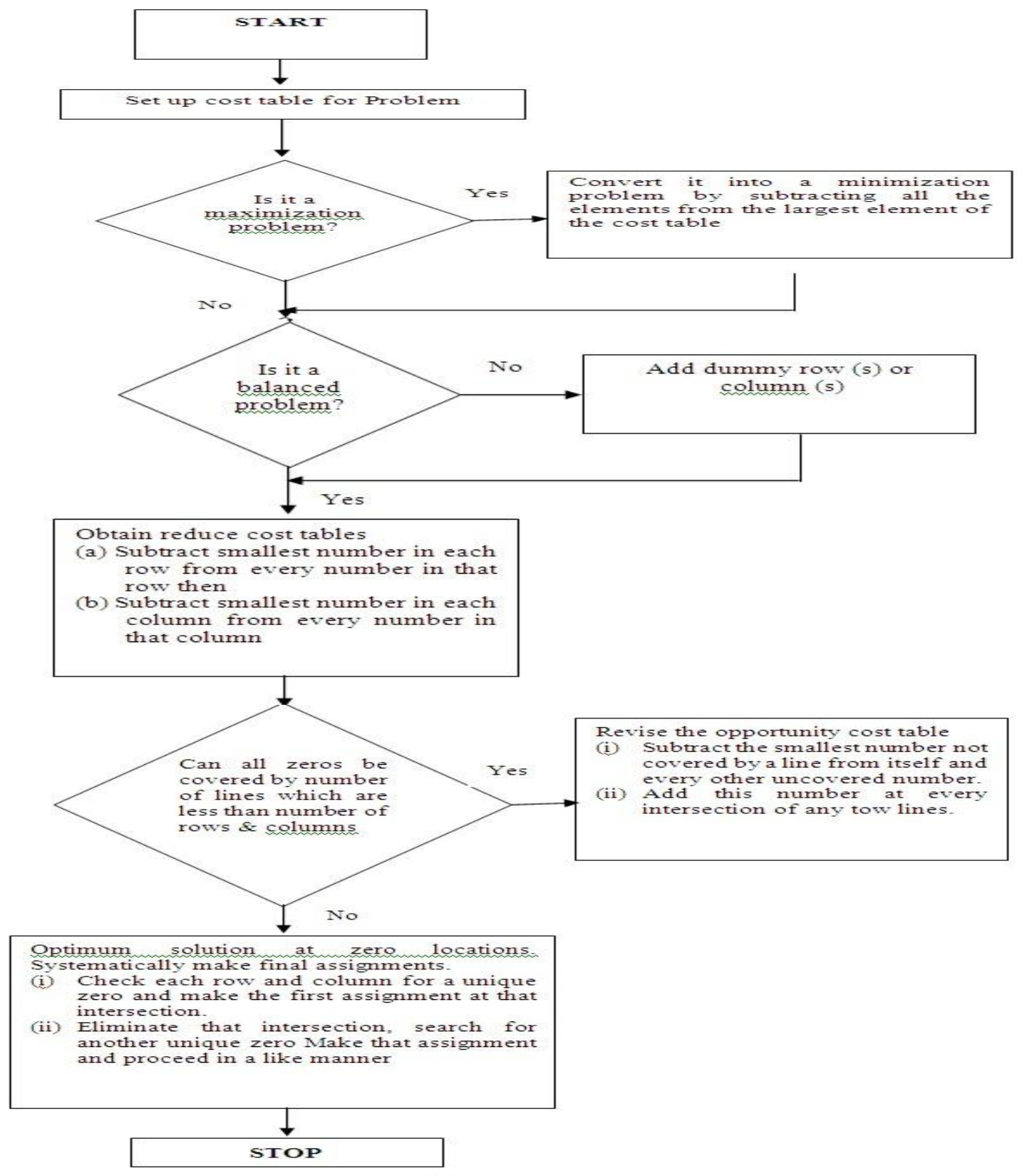

Fig. I flow Chart of steps in the (H-A) method 


\section{MOA- METHOD FOR SOLVING ASSIGNMENT PROBLEM}

This section present a new method to solve the assignment problem which is different from the preceding method. We call it "matrix one's assignment method", because of making assignment in terms of ones.

The new method is based on creating some ones in the assignment matrix and then try to find a complete assignment in terms of ones. By a complete assignment we mean an assignment plan containing exactly $\mathrm{m}$ assigned independent Now, consider the assignment matrix where $\mathrm{C}_{\mathrm{ij}}$ is the cost or effectiveness of assigning. $i^{\text {th }}$ job to $\mathrm{j}^{\text {th }}$ machine.

$$
\left.\begin{array}{llllll}
1 & 2 & 3 & \ldots & n \\
1 & & & & & \\
c_{11} & c_{12} & c_{13} & \ldots & c_{1 n} \\
c_{21} & c_{22} & c_{23} & \ldots & c_{2 n} \\
\vdots & \vdots & \ddots & \vdots \\
c_{n 1} & c_{n 2} & c_{n 3} & \ldots & c_{n n}
\end{array}\right)
$$

The new algorithm is as follows:

Step1. n a minimization (Maximization) case, find the minimum (maximum) element of each row in the assignment matrix $\left(\right.$ say $\mathrm{a}_{\mathrm{i}}$ ) and write it on the right hand side of the matrix.

$$
\left.\begin{array}{cccccc}
1 & 2 & 3 & \ldots . & n \\
2 & & & & & \\
c_{11} & c_{12} & c_{13} & \ldots & c_{1 n} \\
c_{21} & c_{22} & c_{23} & \ldots & c_{2 n} \\
& \vdots & \ddots & \vdots & \vdots \\
& & & & \\
c_{n 1} & c_{n 2} & c_{n 3} & \ldots & c_{n n}
\end{array}\right) \mathrm{a}_{1}
$$

Then divide each element of ith row of the matrix by $\mathrm{a}_{\mathrm{i}}$. These operations create at least one ones in each rows.

In term of ones for each row and column do assignment, otherwise go to step 2.

Step 2. Find the minimum(maximum) element of each column in assignment matrix (say $b_{j}$ ) and write it below jth column. Then divide each element of jth column of the matrix by $b_{j}$.

These operations create at least one ones in each columns. Make assignment in terms of ones. If no feasible assignment can be achieved from step (1) and (2) then go to step 3.

$$
\begin{aligned}
& 1 \\
& 2 \\
& \mathrm{n}
\end{aligned} \quad\left(\begin{array}{ccccc}
1 & 2 & 3 & \ldots \ldots & n \\
c_{11} / a_{1} & c_{12} / a_{1} & c_{13} / a_{1} & \ldots & c_{1 n} / a_{1} \\
c_{21} a_{2} & c_{22} / a_{2} & c_{23} / a_{2} & \ldots & c_{2 n} / a_{2} \\
\vdots & \vdots & \ddots & \vdots \\
c_{n 1} / a_{n} & c_{n 2} / a_{n} & c_{n 3} / a_{n} & \ldots & c_{n 1} / a_{n} \\
b_{1} & b_{2} & b_{3} & \ldots \ldots & b_{n}
\end{array}\right)
$$

Note : In a maximization case, the end of step 2 we have a fuzzy matrix which all elements are belong to $[0,1]$ and the greatest element is one [4]. 
Step 3.Draw the minimum number of lines to cover all the once of the matrix. If the number of drawn lines less than $\mathrm{n}$, then the complete assignment is not possible, which if the number of lines is exactly equal to $\mathrm{n}$, then the complete assignment is obtained.

Step 4. If a complete assignment program is not possible is step 3, then select the smallest (largest) element (say $\mathrm{d}_{i j}$ ) out of those which do not lie on any of the lines in the above matrix. Then divide by $\mathrm{d}_{i j}$ each element of the uncovered rows or columns, which $\mathrm{d}_{i j}$ lies on it. This operation create some new ones to this row or column.

If still a complete optimal assignment is not achieved in this new matrix, then use step 4 and 3 iteratively. By repeating the same procedure the optimal assignment will be obtained.

Priority, plays an important role in this method, when we want to assign the ones.

\section{Priority rule.}

1. For maximization (minimization) assignment problem assign the ones on the rows which have greatest (smallest) element on the right hand side, respectively.

2. One questions arise here, what to do with non square matrix? To make square, a non square matrix, we add one artificial row or column which all elements are one. Thus we solve the problem with the new matrix, by using the new method. The matrix after performing the steps reduces to a matrix which has ones in each rows and columns. So, the optimal assignment has been reached.

\section{Numerical Example Using MOA Method}

Example 2 : Consider the following assignment problem. Assign the five jobs to the three machines so as to minimize the total cost.

1
2
3
4
5 $\left(\begin{array}{lllcc}1 & 2 & 3 & 4 & 5 \\ 7 & 8 & 7 & 15 & 4 \\ 9 & 6 & 1 & 14 & 10 \\ 7 & 6 & 14 & 6 & 10 \\ 9 & 6 & 12 & 10 & 6\end{array}\right)$

1. Find the minimum element of each row in the assignment matrix (say $\mathrm{a}_{i}$ ) and write it on the hand side of the matrix, as follows.

\begin{tabular}{|c|c|c|c|c|c|c|}
\hline & 1 & 2 & 3 & 4 & 5 & \\
\hline 1 & 12 & 8 & 7 & 15 & 4 & 4 \\
\hline 2 & 7 & 9 & 1 & 14 & 10 & 1 \\
\hline 3 & 9 & 6 & 12 & 6 & 7 & 6 \\
\hline 4 & 7 & 6 & 14 & 6 & 10 & 6 \\
\hline 5 & 9 & 6 & 12 & 10 & 6 & 6 \\
\hline
\end{tabular}

Then divide each element of ith row of the matrix by $\mathrm{a}_{i}$. These operations create ones to each rows, and the matrix reduces to following matrix.

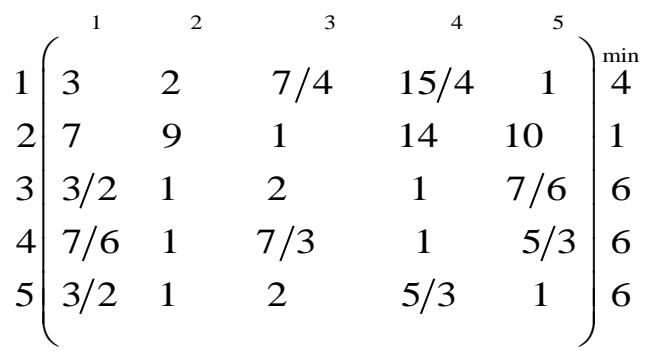


2. Now find the minimum element of each column in assignment matrix (say $b_{j}$ ) and write it below column. Then divide each element of jth column of the matrix by $b_{j}$.

\begin{tabular}{|c|c|c|c|c|c|c|}
\hline & 1 & 2 & 3 & 4 & 5 & \\
\hline 1 & 3 & 2 & $7 / 4$ & $15 / 4$ & 1 & 4 \\
\hline 2 & 7 & 9 & 1 & 14 & 10 & 1 \\
\hline 3 & $3 / 2$ & 1 & 2 & 1 & $7 / 6$ & 0 \\
\hline 4 & $7 / 6$ & 1 & $7 / 3$ & 1 & $5 / 3$ & 6 \\
\hline 5 & $3 / 2$ & 1 & 2 & $5 / 3$ & 1 & 6 \\
\hline & 76 & 1 & 1 & 1 & 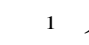 & \\
\hline
\end{tabular}

\begin{tabular}{|c|c|c|c|c|c|c|}
\hline & 1 & 2 & 3 & 4 & 5 & \\
\hline 1 & $18 / 7$ & 2 & $7 / 4$ & $15 / 4$ & 1 & 4 \\
\hline 2 & 6 & 9 & 1 & 14 & 10 & 1 \\
\hline 3 & $18 / 14$ & 1 & 2 & 1 & $7 / 6$ & 6 \\
\hline 4 & 1 & 1 & $7 / 3$ & 1 & $5 / 3$ & 6 \\
\hline 5 & $18 / 14$ & 1 & 2 & $5 / 3$ & 1 & 6 \\
\hline & $7 / 6$ & 1 & 1 & 1 & 1 & \\
\hline
\end{tabular}

3. The minimum number of lines required to pass through all the ones of the matrix is 5 .

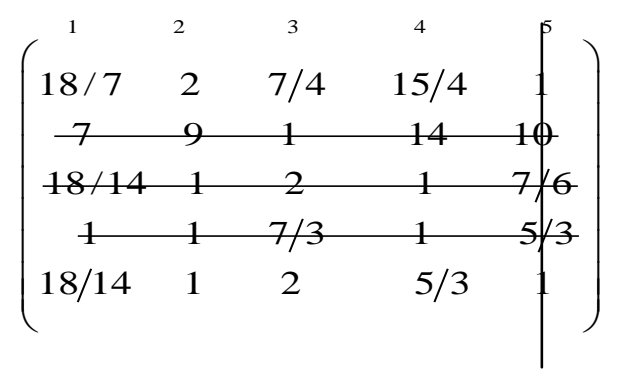

So the complete assignment is possible

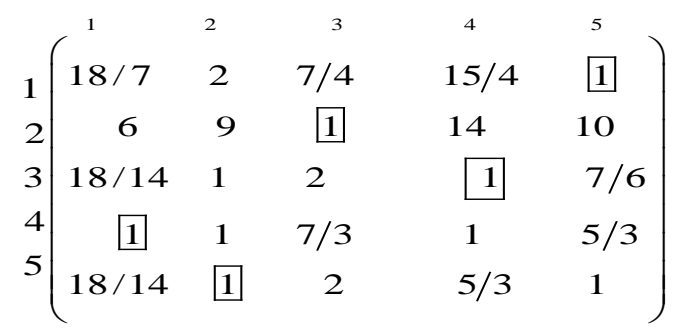

and we can assign the ones and the solution is $(1,5),(2,3),(3,4),(4,1),(5,2)$ and minimum cost is 24 .

We see that cost obtained is 24 using the HA method and MOA- method also the iterations (here steps) required in both the methods is equal. Below the graphical representation is shown comparing the two methods. 


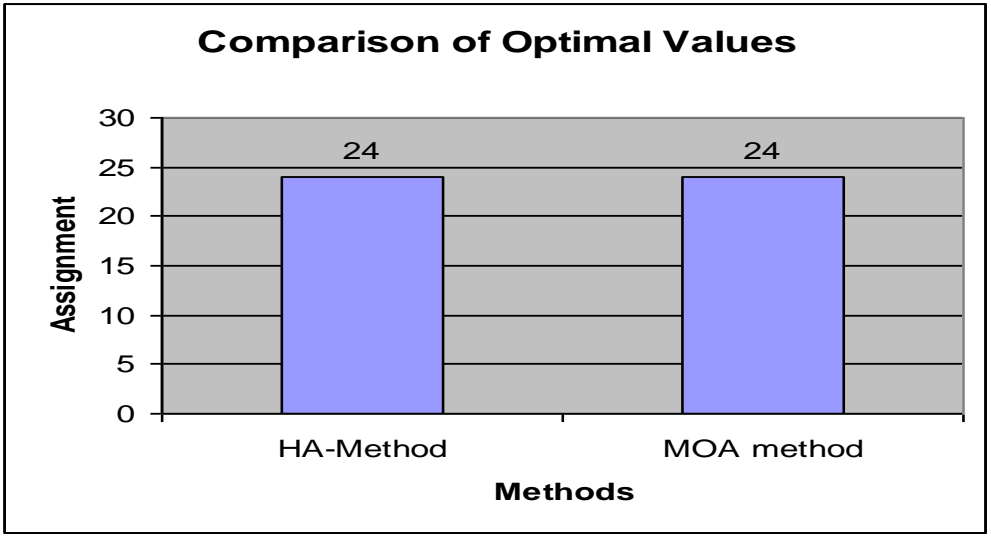

\begin{tabular}{|l|l|l|l|}
\hline Problem & HA-Method & MOA method & Optimum \\
\hline 01 & 24 & 24 & 24 \\
\hline
\end{tabular}

\section{VARIATIONS OF THE ASSIGNMENT PROBLEM}

\section{Non-square matrix (Unbalanced assignment problme):}

Such a problem is found to exist when the number of facilities is not equal to the number of jobs. Since the Hungarian method of solution requires a square matrix, fictious facilities or jobs may be added and zero costs be assigned to the corresponding cells of the matrix . These cells are then treated the same way as the real cost cells during the solution procedure.

Example:-A manufacture of complex electronic equipment has just received a sizable contract and plans to subcontract part of the job. He has solicited bids for 6 subcontracts from 3 firms. Each job is sufficiently large and any firm can take only one job. The table below shows the bids as well as the cost estimates (in lakhs of rupees) for doing the job internally. Not moer than three jobs can be performed internally.

Table-1

\begin{tabular}{|c|c|c|c|c|c|c|}
\hline Firm & 1 & 2 & 3 & 4 & 5 & 6 \\
\hline 1 & 44 & 67 & 41 & 53 & 48 & 64 \\
\hline 2 & 46 & 69 & 40 & 45 & 45 & 68 \\
\hline 3 & 43 & 73 & 37 & 51 & 44 & 62 \\
\hline Internal & 50 & 65 & 35 & 50 & 46 & 63 \\
\hline
\end{tabular}

Find the optimal assignment that will result in minimum total cost

\section{Solution}

Step I. Prepare a Square Matrix :

The problem involves a non-square 4X6 matrix. As three jobs can be performed internally, table 1 (a) may be completed to yield a square matrix

Table 1 (a)

\begin{tabular}{|r|r|l|l|l|l|l|l|}
\hline & & 1 & 2 & 3 & 4 & 5 & 6 \\
\hline & 1 & 44 & 67 & 41 & 53 & 48 & 64 \\
\hline & 2 & 46 & 69 & 40 & 45 & 45 & 68 \\
\hline & 3 & 43 & 73 & 37 & 51 & 44 & 62 \\
\hline & $I_{1}$ & 50 & 65 & 35 & 50 & 46 & 63 \\
\hline & $I_{2}$ & 50 & 65 & 35 & 50 & 46 & 63 \\
\hline & $I_{3}$ & 50 & 65 & 35 & 50 & 46 & 63 \\
\hline
\end{tabular}


Step II. Reduce the Matrix: Performing row and column reductions we get tables 1 (b) and 1 (c) respectively.

Table 1 (b) Matrix after Row reduction

\begin{tabular}{|r|l|l|l|l|l|l|}
\hline & 1 & 2 & 3 & 4 & 5 & 6 \\
\hline 1 & 3 & 26 & 0 & 12 & 7 & 23 \\
\hline 2 & 6 & 69 & 0 & 5 & 5 & 28 \\
\hline 3 & 6 & 36 & 0 & 14 & 7 & 25 \\
\hline $\mathrm{I}_{1}$ & 15 & 30 & 0 & 15 & 11 & 28 \\
\hline $\mathrm{I}_{2}$ & 15 & 30 & 0 & 15 & 11 & 28 \\
\hline $\mathrm{I}_{3}$ & 15 & 30 & 0 & 15 & 11 & 28 \\
\hline
\end{tabular}

Table 1 (c) Matrix after column reduction

\begin{tabular}{|c|l|l|l|l|l|l|}
\hline & 1 & 2 & 3 & 4 & 5 & 6 \\
\hline 1 & 0 & 0 & 0 & 7 & 2 & 0 \\
\hline 2 & 3 & 3 & 0 & 0 & 0 & 5 \\
\hline 3 & 3 & 10 & 0 & 9 & 2 & 2 \\
\hline$I_{1}$ & 12 & 4 & 0 & 10 & 6 & 5 \\
\hline$I_{2}$ & 12 & 4 & 0 & 10 & 6 & 5 \\
\hline$I_{3}$ & 12 & 4 & 0 & 10 & 6 & 5 \\
\hline
\end{tabular}

Step III. Check if Optimal Assignment can be made in the curret Reduced Matrix or not: Draw the minimum number of lines covering all zeros is only $3(<6)$. Hence optimal assignment connot be made in the current feasible solution.

Table- $1(\mathrm{~d})$

\begin{tabular}{|c|c|c|c|c|c|c|}
\hline \multicolumn{7}{|c|}{ 田 } \\
\hline & 1 & 2 & $3_{i}$ & 4 & 5 & 6 \\
\hline 1 & 0 & $\not$ & ) & 7 & 2 & $\not$ \\
\hline 2 & 3 & 3 & te & 70 & ar & 5 \\
\hline 3 & 3 & 10 & d & 9 & 2 & 2 \\
\hline $\mathrm{I}_{1}$ & 12 & 4 & $d$ & 10 & 6 & 5 \\
\hline $\mathrm{I}_{2}$ & 12 & 4 & $d$ & 10 & 6 & 5 \\
\hline $\mathrm{I}_{3}$ & 12 & 4 & $d$ & 10 & 6 & 5 \\
\hline
\end{tabular}

Step IV. Iterate towards an Optimal Solution : We get table 1 (e). Further iterations yield tables 1 (f) and $1(\mathrm{~g})$.
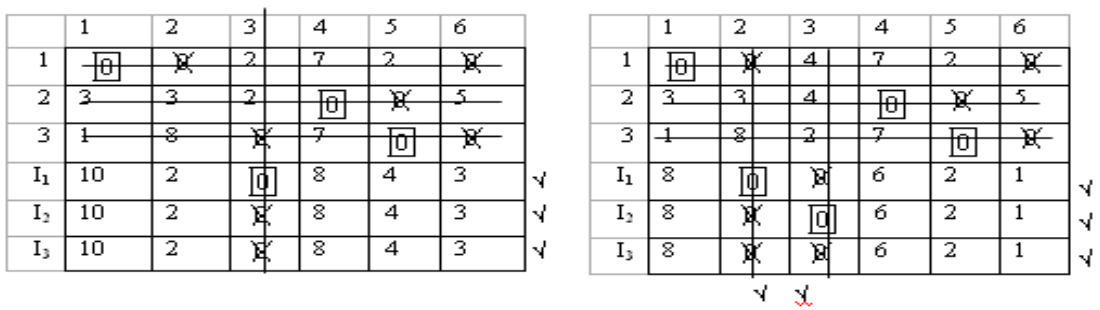

Table $1(\mathrm{~g})$

\begin{tabular}{|c|c|c|c|c|c|c|c|}
\hline & 1 & 2 & 3 & 4 & 5 & 6 & \\
\hline 1 & 0 & 1 & 5 & 7 & 2 & $\mathrm{R}$ & \\
\hline 2 & 3 & 4 & 5 & 0 & $x$ & 5 & \\
\hline 3 & 1 & 9 & 3 & 7 & 0 & \multicolumn{2}{|c|}{$\begin{array}{l}\text { ABBYY FineReader } 11>\times \\
\text { C Start ABBYY FineReader } 11\end{array}$} \\
\hline $\mathrm{I}_{1}$ & 7 & 0 & 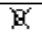 & 5 & 1 & 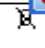 & \\
\hline $\mathrm{I}_{2}$ & 7 & 8 & 回 & 5 & 1 & $g$ & \\
\hline $\mathrm{I}_{3}$ & 7 & 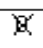 & $x$ & 5 & 1 & 0 & \\
\hline
\end{tabular}

Optima1 Solution 


\section{Optimal Solution}

Table $1(\mathrm{~g})$ gives optimal assignment policy. The associated minimum optimal cost is $(44+45+44+65+35+63)=$ Rs. 296 lakh.

2. Maximization Problem. Sometimes the assignment problem may deal with maximization of the objective function. The maximization problem has to be changed to minimization before the Hungarian method may be applied. This transformation may be done in either of the following two ways:

a. by subtracting all the elements from the largest element of the matrix.

b. by multiplying the matrix elements by-1

Example 2 :- A company has four territories open and four salesmen available for assignment. The territories are not equally rich in their sales potential. It is estimated that a typical salesman operation in each territory would bring in the following annual sales:

Territory

Annual sales (RS)

$$
60,000
$$

III

50,000

III

40,000

IV 30,000

The four salemen are also considered to differ in ability: it is estimated that working under the same conditions, their yearly sales would be proportionately as follows:

\section{Salesman}

Proportion
A B

7
$\mathrm{C}$

$\mathrm{D}$

If the criterion is maximum expected total sales, the intutive answer is to assign the best salesman to the richest territory, the next best salesmen to the second richest territory and so on Verify this answer by the assignment method

\section{Solution}

Step I: Prepare a Square Matrix : The sum of sales of four salesmen $=7+5+5+4=21$. Taking the sale of Rs. 10,000 as one unit of sale, the annual sales of the four salesmen in the four territories are

$\begin{array}{ll}\text { For } & A: \frac{7}{21} \times 6, \times \frac{7}{21} \times 5, \times \frac{7}{21} \times 4, \times \frac{7}{21} \times 3 \\ \text { For } & B: \frac{5}{21} \times 6, \times \frac{5}{21} \times 5, \times \frac{5}{21} \times 4, \times \frac{5}{21} \times 3 \\ \text { For } & C: \frac{5}{21} \times 6, \times \frac{5}{21} \times 5, \times \frac{5}{21} \times 4, \times \frac{5}{21} \times 3 \\ \text { And For } & D: \frac{4}{21} \times 6, \times \frac{4}{21} \times 5, \times \frac{4}{21} \times 4, \times \frac{4}{21} \times 3\end{array}$

In order to avoid the fractional values of annual sales of each salesman in each territory, it will be rather convenient to consider the sales for 21 years. The problem is to determine the assignment which make the total sales maximum, and the effectiveness matrix is given by.

Table 2. (a)

Territrories

Salesmen

\begin{tabular}{l|l|l|l|l|}
\multicolumn{1}{c}{} & \multicolumn{1}{c}{ I } & \multicolumn{1}{c}{ II } & \multicolumn{1}{c}{ III } & \multicolumn{1}{l}{ IV } \\
\cline { 2 - 5 } A & 42 & 35 & 28 & 21 \\
\cline { 2 - 5 } B & 30 & 25 & 20 & 15 \\
\cline { 2 - 5 } C & 30 & 25 & 20 & 15 \\
\cline { 2 - 5 } D & 24 & 20 & 16 & 12 \\
\cline { 2 - 5 } & & &
\end{tabular}




\section{Maximization Problem}

Step II: Convert the Maximization Problem into Minimization. The above maximization problem can be converted into the equivalent minimization problem by subtracting all the matrix elements from the highest elements which is 42 . Table shows the resulting matrix.

Table 2 (b)

Minimization problem

\begin{tabular}{|c|c|c|c|c|}
\hline \multicolumn{2}{|r|}{ I } & II & III & IV \\
\hline A & 0 & 7 & 14 & 21 \\
\hline B & 12 & 17 & 22 & 27 \\
\hline $\mathrm{C}$ & 12 & 17 & 22 & 27 \\
\hline D & 18 & 22 & 26 & 30 \\
\hline
\end{tabular}

Table 2(c)

Matrix after row reduction

I II III IV

\begin{tabular}{l|l|l|l|l|}
\cline { 2 - 5 } A & 0 & 7 & 14 & 21 \\
\cline { 2 - 5 } B & 0 & 5 & 10 & 15 \\
\cline { 2 - 5 } C & 0 & 5 & 10 & 15 \\
\cline { 2 - 5 } D & 0 & 4 & 8 & 12 \\
\cline { 2 - 5 } & &
\end{tabular}

Step III : Reduce the Matrix. Reduction of the matrix 2(b) by the usual method yields tables 2 (c) and 2 (d) Table $2(\mathrm{~d})$

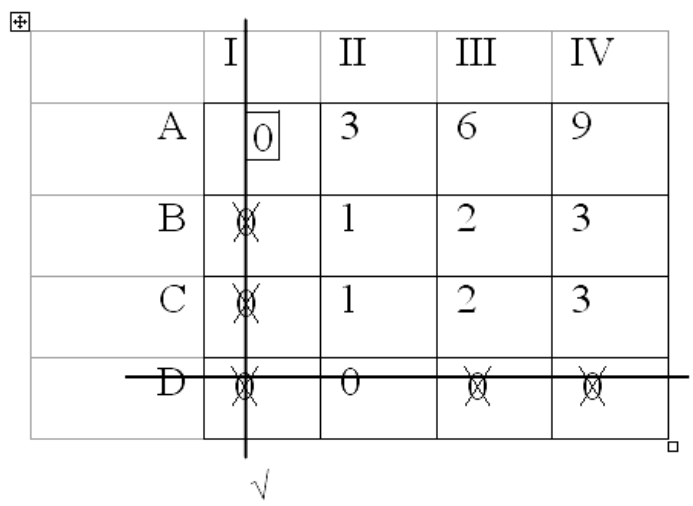

Initial basic feasible solution

Step IV. Check if Optimal Assignment can be made in the Current Feasible Solution or not. Draw the minimmum number of lins covering all zeros are only $2(<4)$. Hence optimal assignment cannot be made in the current feasible solution.

Step V. Iterate towards an Optimal Solution. We proced according to the alogrithm and we get table 2 (e) and thereafter table 2(f), which is optimal.

Table 2 (e)

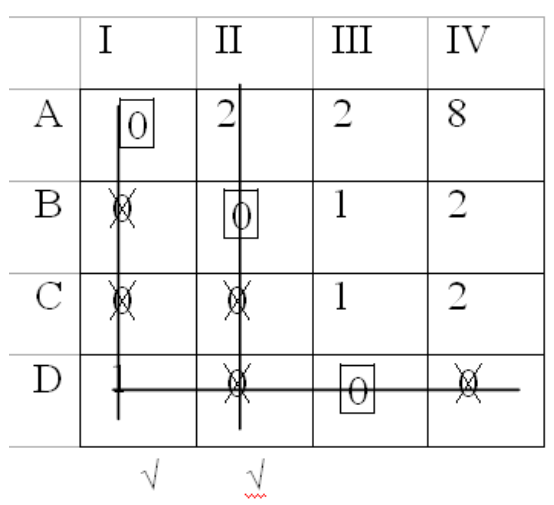

Table 2 (f)

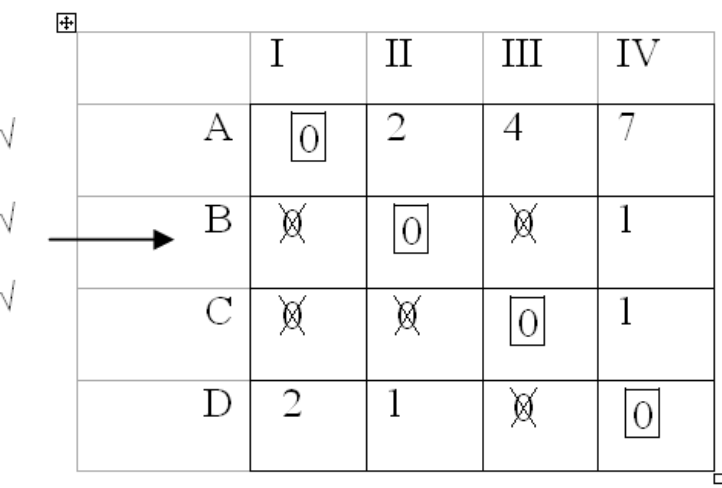


sales are $\frac{1}{21}(42+25+20+12) \times 10,000$

$=$ Rs. $47,142.86$

3. Restrictions on Assignments: Sometime technical, space, legal or other restrictions do not permit the assignment of a particular facility to a particular job. Such problem can be solved by assigning a very heavy cost (infinite cost) to the corresponding cell. Such a job will then be automatically exculed from further consideration (making assignments)

Example 3:- (Assignment Problem- Restrictions on Assignment) Four new machines $\mathrm{M}_{1}, \mathrm{M}_{2}, \mathrm{M}_{3}$ and $\mathrm{M}_{4}$ are to be installed in a machine shop. There are five vacant places A,B,C, D and E available. Because of limited space, machine $\mathrm{M}_{2}$ cannot be place at $\mathrm{C}$ and $\mathrm{M}_{3}$ cannot be placed at $\mathrm{A}$. $\mathrm{C}_{\mathrm{ij}}$ the assignment cost of machine $\mathrm{i}$ to place $\mathrm{j}$ in rupees is shown below.

Table 3 (a)

\begin{tabular}{l|l|l|l|l|l|}
\multicolumn{1}{c}{} & \multicolumn{1}{c}{$\mathrm{A}$} & \multicolumn{1}{c}{$\mathrm{B}$} & $\mathrm{C}$ & $\mathrm{D}$ & \multicolumn{1}{c}{$\mathrm{E}$} \\
\cline { 2 - 7 } $\mathrm{M}_{1}$ & 4 & 6 & 10 & 5 & 6 \\
\cline { 2 - 6 } $\mathrm{M}_{2}$ & 7 & 4 & - & 5 & 4 \\
$\mathrm{M}_{3}$ & - & 6 & 9 & 6 & 2 \\
\cline { 2 - 6 } $\mathrm{M}_{4}$ & 9 & 3 & 7 & 2 & 3 \\
\cline { 2 - 6 } & & & & &
\end{tabular}

Find the optimal assignment schedule

Solution :

Step I. Prepare a Square Matrix. As the given matrix is non-square, we add a dummy machine and associate zero cost with the corresponding cells. As machine $\mathrm{M}_{2}$ cannot be placed at $C$ amd $\mathrm{M}_{3}$ cannot be placed at $\mathrm{A}$, we assign infinite cost $(\infty)$ in cell $\left(\mathrm{M}_{2}, \mathrm{C}\right)$ and $\left(\mathrm{M}_{3} \mathrm{~A}\right)$ resulting in the following matrix.

Table 3 (b)

\begin{tabular}{r|l|l|l|l|l|}
\multicolumn{1}{c}{} & \multicolumn{1}{c}{$\mathrm{A}$} & $\mathrm{B}$ & $\mathrm{C}$ & $\mathrm{D}$ & $\mathrm{E}$ \\
\cline { 2 - 6 } $\mathrm{M}_{1}$ & 4 & 6 & 10 & 5 & 6 \\
\cline { 2 - 6 } $\mathrm{M}_{2}$ & 7 & 4 & $\infty$ & 5 & 4 \\
\cline { 2 - 6 } $\mathrm{M}_{3}$ & $\infty$ & 6 & 9 & 6 & 2 \\
\cline { 2 - 6 } $\mathrm{M}_{4}$ & 9 & 3 & 7 & 2 & 3 \\
\cline { 2 - 6 } $\begin{array}{c}\text { Dummy } \\
\text { machine }\end{array}$ & 0 & 0 & 0 & 0 & 0 \\
\cline { 2 - 6 } & & & & &
\end{tabular}

Step II. Reduce the matrix :

Table 3 (c)

\begin{tabular}{l|l|l|l|l|l|}
\multicolumn{1}{c}{} & \multicolumn{1}{c}{ A } & \multicolumn{1}{c}{ B } & \multicolumn{1}{c}{ C } & D & E \\
\cline { 2 - 6 } $\mathrm{M}_{1}$ & 0 & 2 & 6 & 1 & 6 \\
\cline { 2 - 6 } $\mathrm{M}_{2}$ & 3 & 0 & $\infty$ & 1 & 0 \\
$\mathrm{M}_{3}$ & $\infty$ & 4 & 7 & 4 & 0 \\
$\mathrm{M}_{4}$ & 7 & 1 & 5 & 0 & 1 \\
\cline { 2 - 6 } Dummy & 0 & 0 & 0 & 0 & 0 \\
\cline { 2 - 6 } & & & &
\end{tabular}


Step III. Check if Optimal Assignment can be made in the Current Feasible Solution or not.

Table 3 (d)

\begin{tabular}{|c|c|c|c|c|c|}
\hline & A & B & $\mathrm{C}$ & D & $\mathrm{E}$ \\
\hline $\mathrm{M}_{1}$ & 0 & 2 & 6 & 1 & 2 \\
\hline $\mathrm{M}_{2}$ & 3 & 0 & $\infty$ & 1 & $\not 1$ \\
\hline $\mathrm{M}_{3}$ & $\infty$ & 4 & 7 & 4 & 0 \\
\hline $\mathrm{M}_{4}$ & 7 & 1 & 5 & 0 & 1 \\
\hline Dummy & $\not$ & $\not 1$ & 0 & $\not$ & $\not 1$ \\
\hline
\end{tabular}

As there is no now and no column without assignment optimal assignment can be made in the initial feasible solution. The optimal assignment of various machines is as follows:

Machin $\mathrm{M}_{1}$ to place $\mathrm{A}$,

Machin $\mathrm{M}_{2}$ to place $\mathrm{B}$,

Machin $\mathrm{M}_{3}$ to place $\in$,

Machin $\mathrm{M}_{4}$ to place $\mathrm{D}$,

And place $\mathrm{C}$ will remain vacant.

Total assignment cost $=$ Rs. $(4+4+2+2)=$ Rs 12 .

\section{SPECIAL CASES IN ASSIGNMENT PROBLEM \& ITS FORMULATION}

\section{Formulation of Traveling Salesman Problem (TSP) as an Assignment Problem}

A traveling salesman has to visit $\mathrm{n}$ cities and return to the starting point. He has to start from any one city and visit each city only once. Suppose he starts from the $\mathrm{K}^{\text {th }}$ city and the last city he visited is $\mathrm{m}$. Let the cost of travel fron $\mathrm{i}^{\text {th }}$ city to $\mathrm{j}^{\text {th }}$ city be $\mathrm{c}_{\mathrm{ij}}$. Then the objective function is

Minimize $\sum_{i=1}^{m} \sum_{j=1}^{n} c_{i j} x_{i j}$

Subject to the constraints

$$
\begin{array}{ll}
\sum_{i=1}^{n} x_{i j}=1 & \text { for } \mathrm{i}=1,2, \ldots \mathrm{m}, \mathrm{i} \neq \mathrm{j}, \mathrm{i} \neq \mathrm{m} \\
\sum_{j=1}^{n} x_{i j}=1 & \text { for } \mathrm{j}=1,2, \ldots \mathrm{m}, \mathrm{i} \neq \mathrm{j}, \mathrm{i} \neq \mathrm{n} \\
x_{m k}=1 & \\
x_{i j}=0 \text { or } 1 &
\end{array}
$$

\section{Solution Procedure :-}

Solve the problem as an assignment problem using the method used to solve the above example. It the solutions thus found out are cyclic in nature, then that is the final solution. If it is not cyclic, then select the lowest entry in the table (other than zero). Delete the row and column of this lowest entry and again do the zero assignment in the remaining matrix. Check whether cyclic assignment is available. It not, include the next higher entry in the table and the procedure is repeated until a cyclic assignment is obtained.

The procedure is explained through an example below. 
Example : Consider a four city TSP for which the cost between the city pairs are as shown in the figure below. Find the tour of the salesman so that the cost of travel is minimal.

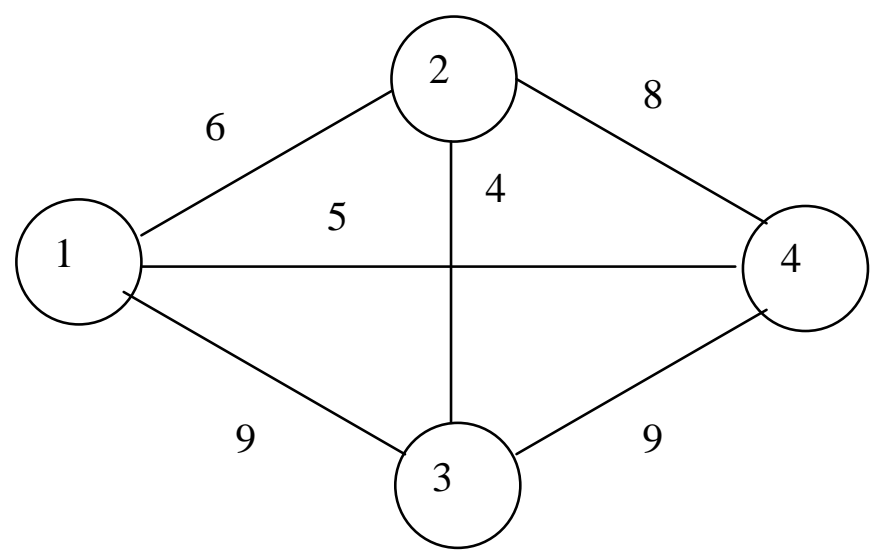

Table 4 (a)

\begin{tabular}{l|cccc|}
1 & $\infty$ & 2 & 3 & 4 \\
\cline { 3 - 5 } 3 & $\infty$ & 4 & 9 & 5 \\
3 & $\infty$ & $\infty$ & 4 & 8 \\
4 & 4 & $\infty$ & 9 \\
5 & 8 & 9 & $\infty$
\end{tabular}

\section{Solution :}

Step 1 : The optimal solution after using the Hungarian method is shown below

\begin{tabular}{c|cccc|}
\multicolumn{5}{c}{ Table 4 (b) } \\
\multicolumn{1}{c}{} & \multicolumn{1}{c}{2} & 3 & 4 \\
\cline { 2 - 5 } 1 & $\infty$ & 0 & 5 & $\underline{0}$ \\
2 & 2 & $\infty$ & $\underline{0}$ & 3 \\
3 & 5 & $\underline{0}$ & $\infty$ & 4 \\
4 & $\underline{0}$ & 3 & 4 & $\infty$ \\
\hline
\end{tabular}

The optimal assignment is $1 \rightarrow 4,2 \rightarrow 3,3 \rightarrow 2,4 \rightarrow 1$ which is not cyclic

Step 2 : Consider the lowest entry ' 2 ' of the cell $(2,1)$. It there is a tie in selecting the lowest eatery, then break the tie arbitraily. Delete the $2^{\text {nd }}$ row and $1^{\text {st }}$ column. Do the zero assignment in the remaining matrix. The resulting table is

Table 4 (c)

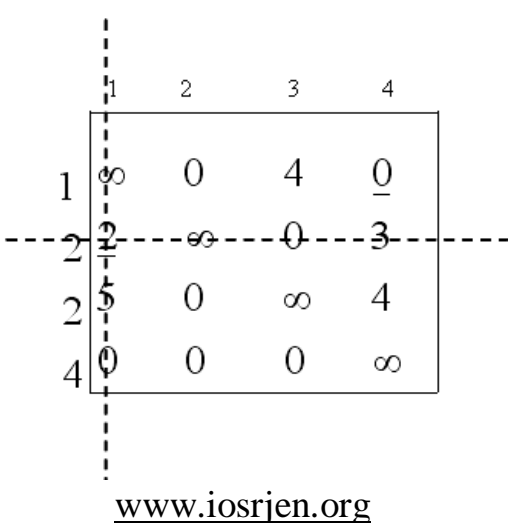


Thus the next optimal assignment assignment is $1 \rightarrow 4,2 \rightarrow 1,3 \rightarrow 2,4 \rightarrow 3$ which is cyclic.

Thus the required tour $1 \rightarrow 4 \rightarrow 3 \rightarrow 2 \rightarrow 1$ and the total travel cost is $5+9+4+6=24$.

\section{CONCLUSION :}

In this paper, a new and simple method was introduced for solving assignment problems. The MOAmethod can be used for all kinds of assignment problems, whether maximize or minimize objective. The new method is based on creating some ones in the assignment matrix and find an assignment in terms of the ones. As cosnsiderable number of methods has been so for presented for assignment problem in which the Hungarian Method is more convenient method among them. Also the comparison between both the methods have been shown in the paper. Therefore this paper attempts to propse a method for solving assignment problem which is different from the preceding methods.

\section{REFERENCES:}

[1] M.S. Bazarra, John J. Jarvis, Hanif D. Sherali, Linear programming and newtwork flows, (2005).

[2] B.S. Goel, S.K. Mittal, Operations REsearch, Fifty Ed., (1982) 2405-2416.

[3] Hamdy A.Taha, Operations Research, an introduction, 8th Ed. (2007).

[4] H.J. Zimmermann, Rudrajit Tapador, Solving the assignment, third Ed. kluwer Academic, Boston, 1996. 